\title{
Influenza vaccination and prevention of cardiovascular disease mortality
}

Catharine Paules and Kanta Subbarao presented in their Seminar (Aug 12, 2017, p 697) the clinical features, therapeutic options, and controversies regarding treatment and prevention of seasonal influenza infection. ${ }^{1}$ Although they acknowledged that influenza can impair different organ systems, little attention was given to cardiovascular 
consequences. Ischaemic heart disease is the leading cause of death worldwide and influenza vaccination could reduce cardiovascular mortality, particularly in patients with coronary heart disease for whom cardiovascular mortality risk has been reported to be halved by influenza vaccination. ${ }^{2}$ Although this conclusion relies on moderate-quality randomised placebo-controlled trials enrolling a small number of patients followed up for up to 1 year, ${ }^{2}$ the possible reduction of cardiovascular disease burden in patients with coronary heart disease is huge.

In Europe, which has an influenza vaccination coverage of $45.6 \%{ }^{3}$ about 30 million individuals have ischaemic heart disease, ${ }^{4}$ and more than half a million of these people will die from cardiovascular causes (figures based on the conservative standardised mortality rates of individuals aged 65 years and older ${ }^{5}$ ). Considering that influenza vaccination is associated with a $24 \%$ risk reduction of cardiovascular mortality in this population (the lower limit of $95 \% \mathrm{Cl}$ of the estimate), ${ }^{4}$ an increase of coverage to $75 \%$ (as recommended by the European Council ${ }^{3}$ ) would translate to about 42000 potentially avoidable cardiovascular deaths in Europe among individuals with ischaemic heart disease.

We think that highlighting this potential effect on ischaemic heart disease is important, since this disease is responsible for $12.8 \%$ of total disability-adjusted life-years lost in Europe, ${ }^{3}$ and thus is relevant for all stakeholders in health-care decision making.

JjF has received speaker and consultant fees from GlaxoSmithKline, Novartis, Teva Pharmaceutical Industries, Lundbeck, Solvay, Abbott, Bial, Merck Serono, Grünenthal, and Merck Sharp and Dohme. $D C$ and JC declare no competing interests.

*Daniel Caldeira, Joaquim J Ferreira, João Costa

dgcaldeira@hotmail.com

Laboratório de Farmacologia Clínica e Terapêutica Instituto de Medicina Molecular, Faculdade de Medicina, Universidade de Lisboa, 1649-028 Lisboa, Portugal
1 Paules C, Subbarao K. Influenza. Lancet 2017; 390: 697-708.

2 Clar C, Oseni Z, Flowers N, Keshtkar-Jahromi M, Rees K. Influenza vaccines for preventing cardiovascular disease. Cochrane Database Syst Rev 2015; 5: CD005050.

3 Mereckiene J. ECDC Technical report. Seasonal influenza vaccination in Europe. Overview of vaccination recommendations and coverage rates in the EU member states for the 2012-13 influenza season. Stockholm: European Centre for Disease Prevention and Control, 2015.

4 Wilkins E, Wilson L, Wickramasinghe K, et al. European cardiovascular disease statistics 2017 edition. Brussels: European Heart Network, 2017.

5 Eurostat Statistics Explained. Causes of deathdiseases of the circulatory system, residents, 2013. http://ec.europa.eu/eurostat/statisticsexplained/index.php/File:Causes_of_ death_\%E2\%80\%94_diseases_of the circulatory_system,_residents,_2013.png (accessed April 4, 2017)

\section{Authors' reply}

We thank Daniel Caldeira, Joaquim J Ferreira, and João Costa for highlighting the effect of influenza infection on underlying cardiovascular disease and the role of influenza vaccination in potentially mitigating this risk. Although we briefly mentioned that influenza infection could exacerbate underlying cardiovascular disease in our Seminar, ${ }^{1}$ we were unable to fully expand on this point because of wordcount restrictions. In this regard, an epidemiological link between influenza and excess deaths associated with cardiovascular disease was suggested as early as in the 1930s. ${ }^{2}$ Since then, several observational studies have supported an association between influenza infection, influenza-like illness, or respiratory tract infections and poor outcomes associated with underlying cardiovascular disease. In particular, a strong association with an increased risk of acute myocardial infarction has been reported. ${ }^{2,3}$ Therefore, influenza vaccination could potentially prevent cardiovascular events; a Cochrane review evaluated evidence for this intervention. ${ }^{4}$ The systematic analysis revealed that in randomised controlled trials that examined influenza vaccination as a mechanism of secondary prevention, significantly fewer cardiovascular deaths occurred in the vaccine group than in the control group (risk ratio 0.45, 95\% Cl 0.26-0.76; $p=0.003$ ). However, a difference was not observed for specific cardiovascular events such as acute myocardial infarction, and the effectiveness of influenza vaccination in primary prevention could not be assessed from available data.

The findings from the Cochrane review had moderate bias because sample sizes were small and cardiovascular events were rare. However, the findings support influenza vaccination in individuals with cardiovascular disease, and are reflected in the recommendations for influenza vaccination from the US Centers for Disease Control and Prevention $^{5}$ and the European Centre for Disease Prevention and Control. ${ }^{6}$ As stated by Caldeira and colleagues in their Correspondence, cardiovascular disease is a leading cause of death and disability worldwide. Mitigating the risk of influenza infection in this highrisk group through interventions such as vaccination could have a substantial positive effect on public health. More studies are needed to fully understand the association between influenza infection and cardiovascular events and the role of influenza vaccination as a prevention strategy.

We declare no competing interests.

\section{Catharine I Paules, *Kanta Subbarao kanta.subbarao@influenzacentre.org}

Office of the Director, National Institute of Allergy and Infectious Diseases, National Institutes of Health, Bethesda, MD, USA (CIP); and WHO Collaborating Centre for Reference and Research on Influenza and Department of Microbiology and Immunology, The University of Melbourne, The Peter Doherty Institute for Infection and Immunity, Melbourne, VIC 3000, Australia (KS)

1 Paules C, Subbarao K. Influenza. Lancet 2017; 390: 697-708.

2 Barnes M, Heywood AE, Mahimbo A, Rahman B, Newall AT, Macintyre CR. Acute myocardial infarction and influenza: a meta-analysis of case-control studies. Heart 2015; 101: 1738-47.

3 Nguyen JL, Yang W, Ito K, Matte TD, Shaman J, Kinney PL. Seasonal influenza infections and cardiovascular disease mortality. JAMA Cardiol 2016; 1: 274-81.

4 Clar C, Oseni Z, Flowers N, Keshtkar-Jahromi M, Rees K. Influenza vaccines for preventing cardiovascular disease. Cochrane Database Syst Rev 2015; 5: CD005050.

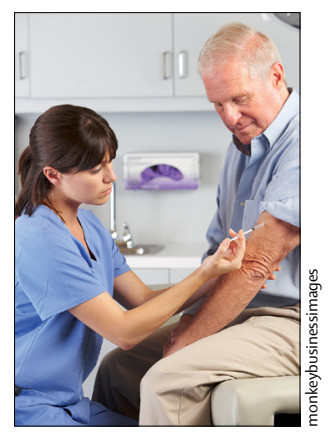

